\title{
Fetal heart rate and uterine contractility during maternal exercise at term
}

\author{
Wilhelmina E.M. Spinnewijn, MD, Frederik K. Lotgering, MD, PhD, Piet C. Struijk, BSc, and \\ Henk C.S. Wallenburg, MD, PhD \\ Rotterdam, The Netherlands
}

OBJECTIVE: Our aim was to assess the physiologic response of human fetal heart rate and uterine contractility to moderately strenuous maternal exercise.

STUDY DESIGN: We measured fetal heart rate and intrauterine pressure with the use of internal monitoring before, during, and after maternal exercise at a heart rate of 140 beats $/ \mathrm{min}$ on a cycle ergometer in 30 term women admitted for elective induction of labor. The fetal heart rate tracings were assessed by three observers and were classified according to Fischer et al. and Nijhuis et al., and the frequency and intensity of uterine contractions were determined.

RESULTS: Fetal outcome was good in all cases. There were no significant differences in Fischer scores between rest, exercise, and recovery periods. The fetuses displayed a heart rate pattern $A$ and $B$, indicative of behavioral states $1 \mathrm{~F}$ or $2 \mathrm{~F}, 85 \%$ of the time, with state changes apparently independent of exercise. Uterine activity increased significantly during the exercise period, with a 5.5 -fold increase in contraction frequency and a fourfold increase in time-pressure integral compared with rest, with rapid recovery after the exercise.

CONCLUSION: Exercise in healthy pregnant women at term does not cause a change in fetal heart rate pattern suggestive of fetal distress or a change in fetal behavioral pattern, but it does significantly increase uterine activity. (AM J OBSTET GYNECOL 1996;174:43-8.)

Key words: Pregnancy, exercise, fetal heart rate, uterine contractions

The fetal heart rate (FHR) response to maternal exercise has been studied repeatedly since Hon and Wohlgemuth ${ }^{\mathrm{l}}$ proposed in 1961 that it could serve as a clinical test for uteroplacental insufficiency. Nonetheless, the question as to what is the normal FHR response to maternal exercise has remained unanswered. This may be attributed to several factors. First, it is difficult to measure FHR accurately during matemal exercise and, second, the physiologic burden imposed by the exercise and the physiologic alterations in fetal behavioral states must be taken into account.

Because of motion artifacts induced by maternal exercise, most authors have reported on FHRs measured not during but before and after exercise ${ }^{1-3}$ Conclusions from this data canot be extrapolated to the exercise period itself, because in sheep most fetal variables are known to return to baseline levels within a few minutes of recovery from even exhausting exercise. ${ }^{4}$. FHR during maternal exercise has usually been studied by means of an external

From the Department of Obstetrics and Gynecology, Erasmus University School of Medicine and Health Sciences.

Received for publication August 2, 1994; revised January 6, 1995; accepted May 15, 1995.

Reprint requests: Fred $K$. Lotgering, $M D, P h D$, Erasmus University, Department of Obstetrics and Gynecology, EE 2283, P.O. Box 1738, 3000 DR Rotterdam, The Netherlands.

Copyright (C) 1996 by Mosby-Year Book, Inc.

$0002-9378 / 96 \$ 5.00+0 \quad 6 / 1 / 66301$ transducer, ${ }^{5-8}$ a method that is sensitive to motion artifacts. Indeed, in one study artifact was demonstrated; FHR was identical to the pedaling frequency on the cycle ergometer or the stepping rate at the treadmill. ${ }^{9}$ Such artifacts might explain the periods of severe fetal bradycardia observed during maternal exercise in several studies. ${ }^{5,7.8}$ However, the occurrence of bradycardia was also reported in 16 of 32 fetuses during or after maternal exercise when B-mode echocardiography was used to study the FHR. ${ }^{10}$ Although this method does not carry the same risk of artifact, the data show that many of the periods of fetal bradycardia were of very short duration and may be described as spikes or variable decelerations rather than as bradycardia. Recently the use of direct fetal monitoring was reported; fetal bradycardia was not observed during 4.5 minutes of maternal exercise at approximately $60 \%$ of maximal aerobic capacity. ${ }^{11}$ In instrumented sheep fetal bradycardia has been occasionally reported in relation to maternal exercise and severe hypoxemia, ${ }^{12}$ but in other studies the FHR was reported unchanged, or slightly increased, without evidence of bradycardia or decelerations, ${ }^{4,}{ }^{13}$ and severe hypoxemia was absent even during exhaustive maternal exercise.

Some women report increased uterine contractility during physical exercise. Uterine contractility has been studied with the use of external tocodynamometry before and after maternal exercise. ${ }^{2}$ However, tocodynamom- 

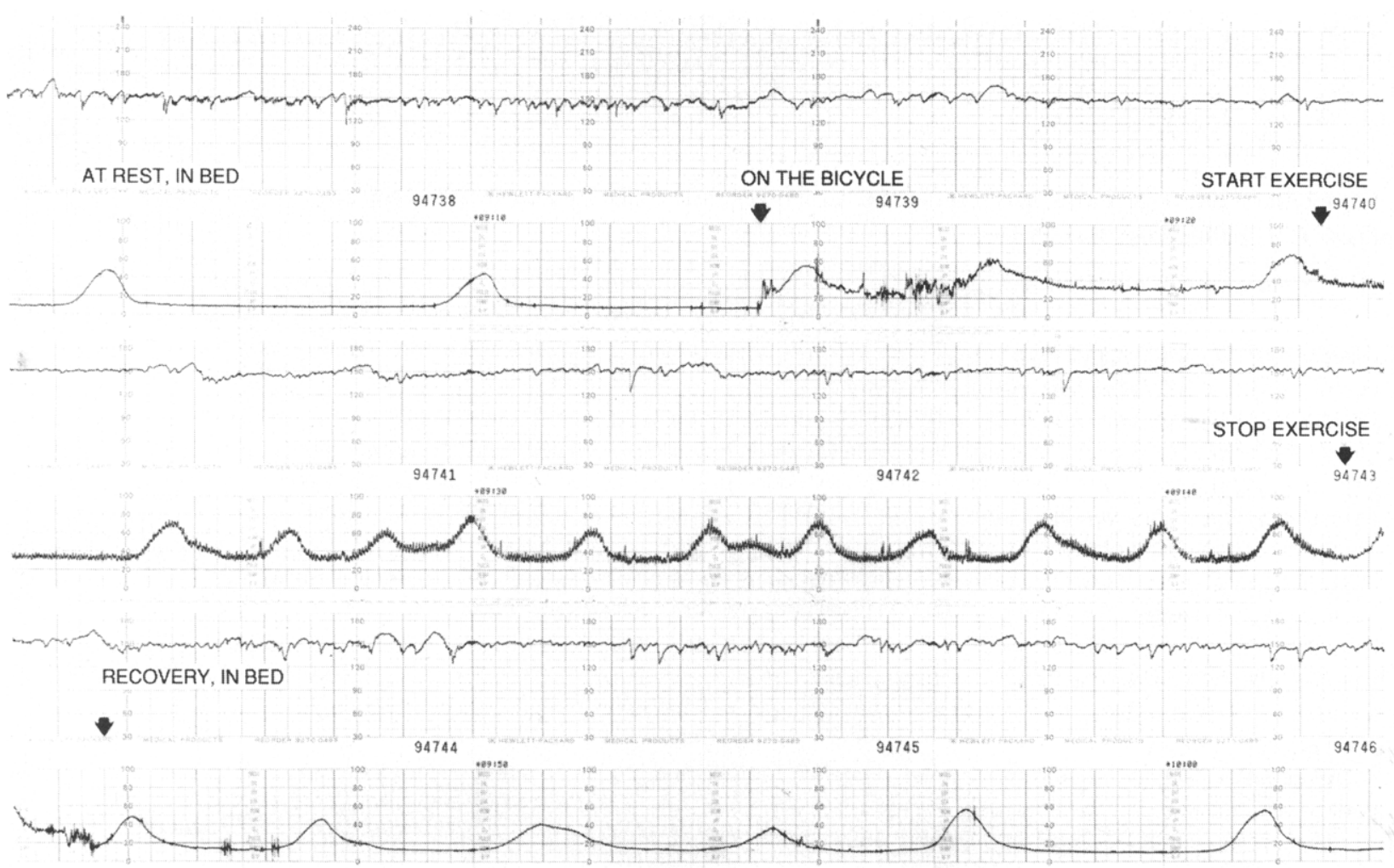

Fig. 1. Typical example of cardiotocogram before, during, and after maternal exercise.

Table I. Fischer scores of FHR before, during, and after maternal exercise

\begin{tabular}{|c|c|c|c|c|}
\hline & \multirow[b]{2}{*}{ Fïscher score } & \multicolumn{3}{|c|}{ No. of feluses } \\
\hline & & Rest & Exercise & Recovery \\
\hline 10 & \multirow{3}{*}{ Optional } & 17 & 13 & 13 \\
\hline 9 & & 6 & 6 & 5 \\
\hline 8 & & 0 & 3 & 3 \\
\hline 7 & & 3 & 2 & 2 \\
\hline 6 & \multirow[t]{2}{*}{ Questionable } & 0 & 1 & 3 \\
\hline 5 & & 0 & 0 & 0 \\
\hline & Critical & 0 & 1 & 0 \\
\hline
\end{tabular}

No significant differences between periods, $n=26$.

etry is rather inaccurate and recovery values provide little information about the effects of exercise itself.

In an effort to determine the physiologic response of the FHR and uterine contractility to maternal exercise, we studied a group of healthy women admitted for elective induction of labor in whom the FHR was recorded from a scalp electrode and intrauterine pressure through an intraamniotic fluid-filled catheter after artificial rupture of the membranes before, during, and after moderately strenuous exercise on the cycle ergometer.

\section{Material and methods}

Between October 1991 and January 1993 we studied 30 healthy pregnant women at a gestational ages between 38 and 42 weeks. All women carried uncomplicated singleton pregnancies with the fetus in the vertex position and the occiput engaged in the pelvis. All women opted for elective induction of labor, which is an accepted procedure in the department, ${ }^{14}$ and had a cervix favorable for induction. The women gave informed consent to participate in the study, which was approved by the hospital and university ethics committee.

The women were admitted at about 8 AM to the airconditioned labor suite, which is kept at $23^{\circ} \mathrm{C}$ and $60 \%$ humidity. The maternal heart rate was continuously recorded and stored as 60-second average values (Polar sport tester, Polar Electro, Kempele, Finland). After artificial rupture of the membranes, a fluid-filied pressure catheter was introduced into the amniotic cavity and an electrode was attached to the fetal scalp; both were connected to an FHR monitor (HP 8040 A, Hewlett-Packard, Boeblingen, Germany). After the pressure transducer was calibrated a 20-minute baseline cardiotocogram was recorded at a paper speed of $3 \mathrm{~cm} \cdot \mathrm{min}^{-1}$, with the woman in the semirecumbent position. Immediately thereafter the woman was seated on the cycle ergometer (Ergoline 900 , Mijnhardt, Bunnik, The Netherlands) placed at her bedside and 5 minutes later she started to pedal at a rate of 60 to 80 revolutions/min and an initial workload of 50 $\mathrm{W}$. The workload was subsequently increased by $10 \mathrm{~W}$ every 30 seconds until a maternal heart rate of approximately 140 beats $\min ^{-1}$ was reached, after which the 
woman continued to exercise for a total of 20 minutes. Immediately after the exercise period the woman returned to the semirecumbent position for recovery. After 20 minutes of recovery she was returned to the care of the attending obstetrician and labor was induced with the use of incremental intravenous doses of oxytocin.

We divided each 60-minute cardiotocogram into three 20-minute periods (baseline, exercise, and recovery) and separated the FHR tracings from the uterine pressure recordings to avoid recognition of the exercise period by the observers. After random numbers were assigned to the 20-minute FHR tracings, the fetal cardiograms were assessed by three independent experienced observers. They classified the cardiograms according to Fischer et al., ${ }^{15}$ as a measure of fetal well-being, and Nijhuis et al., ${ }^{16}$ as a measure of the fetal behavioral state. The tocogram was later reunited with the cardiogram to allow subtyping of decelerations. The Fischer score takes into account the baseline heart rate, variability (band width and zero crossings), accelerations, and decelerations. In this score each variable is assigned a well-defined subscore of 0 (poor), 1 (intermediate), or 2 (good) for each of the five variables, so that the total score ranges from 0 to 10 . Fetal condition is considered critical if the score is $<5$, questionable if the score is between 5 and 7 , and optimal if the score is $\geq 8$. The three observers also classified the cardiotocogram into FHR patterns A, B, C, and D according to Nijhuis et al., which reflect fetal behavioral states $1 \mathrm{~F}$ to $4 \mathrm{~F}$. FHR pattern A has a small band width and isolated accelerations; it is associated with behavioral state $\mathrm{IF}$, characterized by incidental body movements in the absence of eye movements. FHR pattern $B$ has a wider band width and frequent accelerations; it is associated with behavioral state $2 \mathrm{~F}$, characterized by periodic movements in the presence of eye movements. FHR pattern $\mathrm{C}$ also has a wider band width but no accelerations; it is associated with behavioral state $3 \mathrm{~F}$, characterized by absence of body movements in the presence of eye movements. FHR pattern $\mathrm{D}$ has large and long-lasting accelerations; it is associated with behavioral state $4 \mathrm{~F}$, characterized by continuous body and eye movements. We present the median values of Fischer scores and subscores and Nijhuis patterns assigned by the three observers. One observer assessed all 20-minute tocograms with the use of an $x y$-data tablet (Summagraphics, Fairfield, Conn.) and measured the frequency, basal pressure, peak pressure, and time pressure integral of contractions for each period and calculated peak minus basal pressures.

We used the median value and range for each variable under consideration for descriptive statistics. We used SPSS / PC + V 5.02 (SPSS, Chicago) to perform the Friedman and Wilcoxon rank-sign tests to analyze differences between paired variables and Fisher's exact test for differences in behavioral states between periods. A $p$ value $<0.05$ was taken as the level of significance.

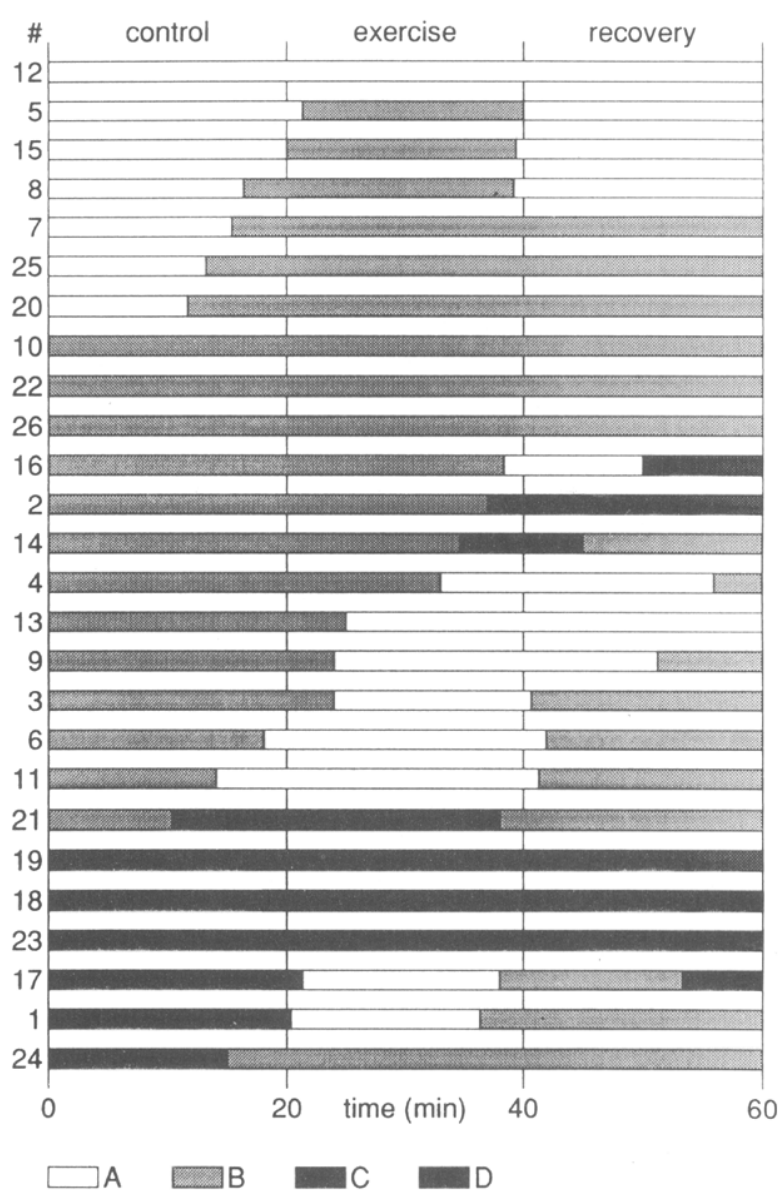

Fig. 2. Effect of moderately strenuous maternal exercise on FHR patterns $A$ to $D$, representing fetal behavioral states $1 F$ to $4 \mathrm{~F}$, after Nijhuis et al. ${ }^{16}$

\section{Results}

Of the 30 women enrolled in the study, six were nulliparous and 24 multiparous; maternal age was 31 (23 to 37) years. Twenty-six women completed the exercise test. Two volunteers withdrew because they found the test too demanding, one woman had strong uterine contractions and decided to stop after 14 minutes of exercise, and in one participant the scalp electrode became detached. We report on the cardiotocograms of the 26 volunteers who completed the test.

Obstetric complications did not occur within 3 hours after the exercise test, and all 30 women were delivered vaginally of healthy infants, 16 boys and 14 girls, with a median 1-minute Apgar-score of 9 (range 4 to 10), a median 5-minute Apgar score of 10 (range 7 to 10), and a median birth weight of $3620 \mathrm{gm}$ (2690 to $4800 \mathrm{gm}$ ). Twenty-seven women had a spontaneous delivery and three were assisted by vacuum extraction $(n=2)$ or by forceps $(n=1)$ because of failure to progress. The maternal heart rate was 92 (79 to 116 ) beats $\cdot \mathrm{min}^{-1}$ at rest and increased to a peak value of 140 (130 to 152 ) beats $\cdot \mathrm{min}^{-1}$ during exercise. 
Table II. FHR before, during, and after maternal exercise

\begin{tabular}{|c|c|c|c|c|c|c|c|c|c|}
\hline \multirow[b]{3}{*}{ Fischer subscore } & \multicolumn{9}{|c|}{ No. of fetuses } \\
\hline & \multicolumn{3}{|c|}{ Rest } & \multicolumn{3}{|c|}{ Exercise } & \multicolumn{3}{|c|}{ Recovery } \\
\hline & 2 & 1 & 0 & 2 & 1 & 0 & 2 & 1 & 0 \\
\hline Basal heart rate & 26 & 0 & 0 & 22 & 4 & 0 & 23 & 3 & 0 \\
\hline Band width & 23 & 3 & 0 & 23 & 3 & 0 & 21 & 5 & 0 \\
\hline Zero crossings & 26 & 0 & 0 & 25 & 1 & 0 & 24 & 2 & 0 \\
\hline Accelerations & 24 & 1 & 1 & 20 & 0 & $6^{*}$ & 21 & 1 & 4 \\
\hline Decelerations & 22 & 3 & 1 & 20 & 6 & 0 & 20 & 6 & 0 \\
\hline
\end{tabular}

$* p<0.05$ compared with rest, $n=26$.

Table III. Uterine contractility before, during, and after maternal exercise

\begin{tabular}{|c|c|c|c|}
\hline Contractions & Rest & Exercise & Recovery \\
\hline Frequency (No. $/ 20 \mathrm{~min}$ ) & $2(0-7)$ & $11(6-18)^{*}$ & $3(0-10)^{*}$ \\
\hline Basal pressure $(\mathrm{mm} \mathrm{Hg})$ & $10(1-20)$ & $28(2-39)^{*}$ & $10(2-24)$ \\
\hline Peak pressure (mm Hg) & $25(3-69)$ & $47(11-70) *$ & $30(5-61)$ \\
\hline (Peak-basal) pressure (mm Hg) & $15(0-57)$ & $15(8-47)$ & $19(0-52)$ \\
\hline Time pressure integral ( $\mathrm{mm} \mathrm{Hg} /$ contraction) & $7(0-39)$ & $9(4-31)$ & $12(0-31)$ \\
\hline Time pressure integral $(\mathrm{mm} \mathrm{Hg} / 20 \mathrm{~min})$ & $25(0-136)$ & $94(46-343) *$ & $38(0-188)$ \\
\hline
\end{tabular}

Median values with range in parentheses.

$* p<0.05$ compared with control values at rest, $n=25$.

A typical example of a cardiotocogram before, during, and after maternal exercise is shown in Fig. 1. Maternal exercise did not significantly affect the basal FHR, which was 140 (130 to 160 ) beats $\cdot \mathrm{min}^{-1}$ at rest, 145 (130 to 170 ) beats $\cdot \min ^{-1}$ during exercise, and 145 (130 to 170 ) beats $\cdot \min ^{-1}$ during recovery. As shown in Table $\mathrm{I}$, the Fischer score at rest was optimal $(\geq 8)$ in 23 and questionable $(5$ to 7 ) in 3 fetuses. There were no significant differences in Fischer scores between periods. One fetus had a "critically" low Fischer score of 4 during exercise; at rest and during recovery the Fischer scores were 7 and 6 , respectively, and 3 hours after the exercise a healthy $3740 \mathrm{gm}$ girl was born with Apgar scores of 9 and 10 after 1 and 5 minutes, respectively. As shown in Table II, significantly more fetuses had no heart rate accelerations during exercise than at rest or during recovery; no significant differences between periods were observed for the other criteria on which the Fischer score is based.

When we assessed FHR patterns according to Nijhuis et al., ${ }^{16}$ at the onset of the recording heart rate pattern $A$ was present in $7, \mathrm{~B}$ in 13, $\mathrm{C}$ in 2 , and $\mathrm{D}$ in 4 fetuses. Fig. 2 shows that the heart rate pattern changed 10 times while the mother was still at rest, 15 times during exercise, and 11 times during recovery. A dominant pattern $\mathrm{A}, \mathrm{B}, \mathrm{C}$, and $D$ was present at rest in $7,13,3$, and 3 cases; during exercise in $9,14,2$, and 1 cases; and during recovery in 9 , 14, 2, and 1 cases, respectively. The changes in FHR patterns occurred in apparently random order, without clustering of (state) changes at the transitions from rest to exercise or from exercise to recovery and without significant differences between periods.
The effect of exercise on uterine contractility is shown in Table III. We report on 25 pressure recordings because in one case the pressure transducer failed. Exercise was associated with a significant increase in the frequency of contractions, from 2 ( 0 to 7 ) contractions per 20-minute period at rest to 11 (6 to 18 ) contractions per 20-minute period during exercise. During recovery the frequency of $3(0$ to 10$)$ contractions per 20 -minute period was not different from that at rest. We did not change the position of the pressure transducer when the volunteer moved from bed to ergometer and vice versa. The estimated position of the catheter tip was approximately $25 \mathrm{~cm}$ higher with the woman on the ergometer than on the bed, which explains why both basal and peak pressures were increased significantly by $18 \mathrm{~mm} \mathrm{Hg}$ during exercise compared with the control period at rest. Peak minus basal pressure differences were not significantly different among periods. Because of the increase in contraction frequency during exercise in the absence of a significantly different time pressure integral of a contraction, the total time pressure integral was significantly higher during the exercise period than at rest or during recovery.

\section{Comment}

Our aim was to describe the physiologic responses of FHR and uterine contractility to moderately strenuous maternal exercise. Because the response to exercise may vary with the type, intensity, and duration of exercise, we controlled for these variables in accordance with the recommendations of the American College of Obstetricians 
and Gynecologists ${ }^{17}$ and imposed cycle exercise at a target heart rate of 140 beats/min, which corresponds to approximately $70 \% \dot{\mathrm{VO}}_{2 \max }$, for 20 minutes, including warming up. Recently, less restrictive American College of Obstetricians and Gynecologists recommendations have been published. ${ }^{18}$ The results from our study should not be extrapolated beyond the limits that we set out (i.e., not to women who are less advanced in their pregnancy or to women who perform exercise of a different type or at a more strenuous level).

In an attempt to differentiate between healthy and compromised fetuses on the basis of the heart rate pattern, Fischer et al. described a score based on five criteria with 0 to 2 points each, which add up to a total score between 0 and 10 points. ${ }^{15}$ To limit the effect of the large interobserver and intraobserver variation in the interpretation of scoring cardiotocograms, ${ }^{19}$ we used the median value of the subscores assigned by three experienced observers to describe each period in each volunteer. Only in one of the 26 cases did we observe an FHR pattern during the exercise that could suggest fetal hypoxemia in a fetus that was born in good health 3 hours later. We did not observe a single case of fetal bradycardia during exercise or recovery. As mentioned in the introduction, some of the observations of fetal bradycardia during exercise reported in the literature may have resulted from artifact or from incorrect nomenclature. It seems unlikely that the absence of fetal bradycardia in our study, in contrast to others, ${ }^{5,7,8,10}$ could have resulted from differences in the duration or intensity of exercise.

Many women report that they feel their baby moving less when they exercise. This could indicate either a real change in fetal movements or a subjective impression. To the best of our knowledge, fetal behavioral states have not been studied in relation to maternal exercise. Heart rate patterns $\mathrm{A}$ to $\mathrm{D}$ describe the fetal behavioral state rather than the state of well-being per se. In accordance with Nijhuis et al., ${ }^{16}$ we observed predominantly patterns A $(27 \%)$ and $\mathrm{B}(50 \%)$ at rest. In spite of the significant increase during exercise in the number of fetuses without heart rate accelerations, the changes in FHR patterns occurred in an apparently random order, as shown in Fig. 2. During exercise the dominant heart rate patterns remained $\mathrm{A}(35 \%)$ and $\mathrm{B}(54 \%)$, corresponding with behavioral states $1 \mathrm{~F}$ and $2 \mathrm{~F}$. This suggests that the changes in fetal movements that women may experience during exercise do not reflect a consistent change in the fetal behavioral pattern but rather a subjective impression. This impression may be caused by distraction or by increased awareness of fetal quiescence as an otherwise normal state change.

Some women report an increase in the number of uterine contractions during physical exercise. Again, this may indicate either a true increase in uterine contractility or a subjective impression. External tocodynamometry has been used to study uterine contractility during ${ }^{20}$ and after $^{2}$ exercise. It was reported that during cycling $50 \%$ of women had uterine contractions, ${ }^{20}$ but, because uterine activity at rest was not mentioned, the extent to which the exercise affected myometrial activity cannot be derived from this study. During recovery from exercise uterine activity was reported unchanged compared with the control period at rest. ${ }^{2}$ However, our study shows that with regard to uterine contractility the recovery period is not representative of the exercise period itself. We found that the peak minus basal pressure difference was unaffected by exercise, so that the intensity of uterine contractions was unaltered by the physical activity. Uterine contractility during exercise at term was increased as a result of a 5.5-fold higher frequency of contractions, associated with a fourfold higher time-pressure integral. During recovery the contraction frequency was still 1.5-fold higher than in the control period. The slightly increased number of contractions during the recovery period may have resulted from the preceding exercise, but it could also be caused by the rupture of the membranes 40 to 60 minutes earlier.

The mechanisms that cause the increase in uterine contractility during the exercise period and restrict it largely to that period are unknown. It might be speculated that some hormonal mechanism may be the cause, but mechanical stimulation of the uterus during movement might also explain the sudden onset and end of uterine contractions during exercise. Our data confirm the impression that exercise indeed increases uterine contractility. However, the results of this study should not be extrapolated to women in whom the uterus is less likely to respond to various stimuli than in our study in term pregnant women admitted for elective induction of labor with a ripe cervix (i.e., to preterm women with an unripe cervix).

We thank J.G. Santema, MD, and A.J. Schneider, MD, for their help with assessment of the cardiotocograms.

\section{REFERENCES}

1. Hon EH, Wohlgemuth $\mathrm{R}$. The electronic evaluation of fetal heart rate, IV: the effect of maternal exercise. Aм J Oвsтет GWNECOL 1961;81:361-71.

2. Veille JC, Hohimer AR, Burry K, Speroff L. The effect of exercise on uterine activity in the last eight weeks of pregnancy. Am J Obstet GyneCOL 1985;151:727-30.

3. Hauth JC, Gilstrap LC, Widmer K. Fetal heart rate reactivity before and after maternal jogging during the third trimester. Am J OBstet GNecol 1981;142:545-7.

4. Lotgering FK, Gilbert RD, Longo LD. Exercise responses in pregnant sheep: blood gases, temperatures, and fetal cardiovascular system. J Appl Physiol 1983;55:842-50.

5. Artal R, Romem Y, Paul RH, Wiswell R. Fetal bradycardia induced by maternal exercise. Lancet 1984;2:258-60.

6. Artal R, Rutherford S, Romem Y, Kammula RK, Dorey FJ, Wiswell RA. Fetal heart rate responses to maternal exercise. AM J OBSTET GVNNECOL 1986;155:729-33.

7. Jovanovic L, Kessler A, Peterson CM. Human maternal and 
fetal response to graded exercise. J Appl Physiol 1985;58 1719-22.

8. Dale E, Mullinax KM, Bryan DH. Exercise during pregnancy: effects on the fetus. Can J Appl Sport Sci 1982;7:98103.

9. Paolone AM, Shangold M, Paul D, Minnitti J, Weiner S. Fetal heart rate measurement during maternal exercise-avoidance of artefact. Med Sci Sports Exerc 1987;19:605-9.

10. Carpenter MW, Sady SP, Hoegsberg B, et al. Fetal heart rate response to maternal exertion. JAMA 1988;259:3006-9.

11. Artal R, Khodiguian N, Paul RH. Intrapartum fetal heart rate responses to maternal exercise: case reports. J Perinat Med 1994;22:499-502.

12. Emmanouilides GC, Hobel CJ, Yashiro K, Klyman G. Fetal responses to maternal exercise in the sheep. AM J OвsteT GYNECOL 1972;112:130-7.

13. Clapp JF III. Acute exercise stress in the pregnant ewe. AM J OBSTET GiNeCOL 1980;136:489-94.

14. Vierhout ME, Out JJ, Wallenburg HCS. Elective induction of labor: a prospective clinical study, I: obstetric and neonatal effects. J Perinat Med 1985;13:155-62.

15. Fischer WM, Stude I, Brandt H. Ein Vorschlag zur Beurtei- lung des antepartualen Kardiotokogramms. Z Geburtshilfe Perinat 1976;180:117-23.

16. Nijhuis JG, Prechtl HFR, Martin CB, Bots RSGM. Are there behavioural states in the human fetus? Early Hum Dev 1982; 6:177-95.

17. American College of Obstetricians and Gynecologists. Exercise during pregnancy and the postnatal period. In: Artal Mittelmark R, Wiswell RA, Drinkwater BL, eds. Exercise in pregnancy. 2nd ed. Baltimore: Williams \& Wilkins, 1991: 313-9.

18. American College of Obstetricians and Gynecologists. Exercise during pregnancy and the postpartum period. Washington, DC: American College of Obstetricians and Gynecologists, 1994. (Technical bulletin no 189.)

19. Lotgering FK, Wallenburg HCS, Schouten HJA. Interobserver and intraobserver variation in the assessment of antepartum cardiotocograms. Am J OBstet GineCOL 1982;144: $701-5$.

20. Durak EP, Jovanovic L, Peterson CM. Comparative evaluation of uterine response to exercise on five aerobic machines. AM J OBSTET GHecol 1990;162:754-6.

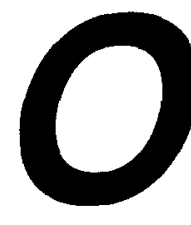

Don't miss a single issue of the journal! To ensure prompt service when you change your address, please photocopy and complete the form below.

Please send your change of address notification at least six weeks before your move to ensure continued service. We regret we cannot guarantee replacement of issues missed due to late notification.

JOURNAL TITLE:

Fill in the title of the journal here.

\section{OLD ADDRESS:}

Affix the address label from a recent issue of the journal here.

\section{NEW ADDRESS:}

Clearly print your new address here.

Name

Address

City/State/ZIP
COPY AND MAIL THIS FORM TO: Journal Subscription Services Mosby-Year Book, Inc. 11830 Westline Industrial Dr. St. Louis, MO 63146-3318
OR PHONE:

1-800-453-4351

Outside the U.S., call

314-453-4351 